\title{
Rapid-Acting Insulin Analogues Versus Regular Human Insulin: A Meta-Analysis of Effects on Glycemic Control in Patients with Diabetes
}

\author{
Antonio Nicolucci - Antonio Ceriello · Paolo Di Bartolo • \\ Antonella Corcos · Marco Orsini Federici
}

Received: June 21, 2019 / Published online: December 23, 2019

(C) The Author(s) 2019

\section{ABSTRACT}

Introduction: The aim of this meta-analysis was to investigate the impact of rapid-acting insulin analogues (RAIAs) and regular human insulin (RHI) on glycemic control, including long- and short-term glycemic variability as

Enhanced Digital Features To view enhanced digital features for this article go to https://doi.org/10.6084/ m9.figshare.10282829.

Electronic Supplementary Material The online version of this article (https://doi.org/10.1007/s13300019-00732-w) contains supplementary material, which is available to authorized users.

\section{A. Nicolucci $(\bowtie)$}

Center for Outcomes Research and Clinical Epidemiology (CORESEARCH), Pescara, Italy e-mail: nicolucci@coresearch.it

\section{A. Ceriello}

Institut d'investigaciones Biomèdiques August Pi

Sunyer (DIBAPS), Barcelona, Spain

A. Ceriello

IRCCS MultiMedica, Milan, Italy

P. Di Bartolo

Direttore UO di Diabetologia, Rete Clinica di

Diabetologia Aziendale - Dipartimento, Internistico

di Ravenna - AUSL Romagna, Ravenna, Italy

A. Corcos - M. Orsini Federici

Eli Lilly Italia S.p.A., Medical Affairs Diabetes, Sesto

Fiorentino, Italy measured by glycated haemoglobin (HbA1c) and pre- and postprandial glucose (PPG).

Methods: PubMed was searched for studies published between 1999 and 29 June 2016. Randomised controlled trials of patients with diabetes that assessed the effects of RAIAs or RHI on glycemic control, focusing on preprandial glucose, PPG and HbA1c, were included. Only studies that reported both means and standard deviations for those outcomes were analysed; from these data, weighted mean differences and $95 \%$ confidence intervals were generated to yield overall point estimates. The primary outcomes of the meta-analysis were the mean differences between RAIAs and RHI at the end of the study in PPG, preprandial glucose, and HbA1c.

Results: Twenty-seven studies $(n=7452)$ were included. The difference in PPG between RAIAand RHI-treated patients was significant-in favour of RAIAs - in patients with type 1 diabetes (T1D) [- $22.2 \mathrm{mg} / \mathrm{dL} ; 95 \%$ confidence interval $(\mathrm{CI})-27.4,-17.0 \mathrm{mg} / \mathrm{dL} ; P<0.0001]$ but not in those with type 2 diabetes (T2D). For preprandial glucose, there was a non-significant trend favouring RHIs in T1D; no data were available for patients with T2D. In patients with $\mathrm{T} 1 \mathrm{D}$, the between-group difference in end-oftreatment (EOT) HbA1c favoured RAIAs $(-0.13 \% ; \quad 95 \% \quad$ CI $-0.18, \quad-0.08 \%$; 
$P<0.0001$ ), but was not significant in patients with T2D. The main study limitations were the small number and heterogeneity of the included studies.

Conclusions: These results demonstrate that RAIAs are more effective at reducing PPG and improving HbA1c than RHIs in T1D. More data are required to assess the effect of these agents on glucose control in T2D.

Plain Language Summary: In patients with diabetes, the risk of complications is increased by poor control of blood glucose levels and high blood glucose variability. Complications may include cardiovascular disease, eye problems and amputation. Control and variability of blood glucose levels can be evaluated using a range of measures, including (i) glycated haemoglobin (HbA1c) level at the end of the treatment period; (ii) change in $\mathrm{HbA1c}$ level during the treatment period; (iii) fasting plasma glucose level; (iv) postprandial glucose (PPG) level; (v) change in blood glucose level after a meal. PPG levels following a meal are an important measure of overall metabolic control in diabetes, and reduction of glycemic variability $(\mathrm{GV})$ can be achieved via reductions in PPG. Both rapid-acting insulin analogues (RAIAs; aspart, glulisine and lispro) and regular human insulin (RHI) are widely used in the management of diabetes. Using data from 27 randomised controlled trials involving more than 7000 patients, we investigated the impact of RAIAs and RHI on measures of glycemic control and variability in patients with type 1 diabetes (T1D) or type 2 diabetes (T2D). Our results show that, in patients with T1D, RAIAs are more effective than RHI at reducing PPG excursions and HbA1c. This indicates that glycemic control is better with RAIAs than with RHI. More data are required to assess the effects of RAIAs and RHI on glycemic control and variability in patients with T2D.

Keywords: Diabetes; Glycemic variability; Meta-analysis; Rapid-acting insulin analogues; Regular human insulin

\section{Key Summary Points}

Suboptimal glycemic control and high glycemic variability $(\mathrm{GV})$ are associated with increased risk of complications in patients with diabetes.

The aim of this meta-analysis was to investigate the impact of rapid-acting insulin analogues (RAIAs) and regular human insulin (RHI) on glycemic control, including long- and short-term GV as measured by end-of-treatment glycated haemoglobin (HbA1c) level, change from baseline in $\mathrm{HbA1c}$, pre- and post-prandial glucose levels, and pre- to postprandial change in blood glucose levels.

Twenty-seven studies involving more than 7000 patients were included in the analysis, which found that RAIAs are more effective than RHI at reducing postprandial glucose and improving HbA1c in patients with type 1 diabetes.

More data are required to assess the relative effects of RAIAs and RHI on glucose control in patients with type 2 diabetes.

\section{INTRODUCTION}

The three main components of dysglycaemia in patients with diabetes are chronic hyperglycaemia, hypoglycaemia and glycemic variability (GV) [1]. GV is a measure of changes in blood glucose (BG) levels, both throughout the day and over time. Long-term GV is most frequently evaluated by fluctuations over weeks or months in glycated haemoglobin (HbA1c), fasting plasma glucose (FPG) and/or postprandial glucose (PPG), while short-term GV involves the measurement of acute (within-day) glucose fluctuations [1, 2]. The most obvious example of these is the change in BG levels after a meal (PPG). PPG levels are an important factor in 
terms of overall metabolic control in diabetes, with some studies finding a closer association between PPG levels and HbA1c than between FPG levels and HbA1c [3, 4]. Reducing postprandial excursions is a valuable strategy for reducing $\mathrm{GV}$ in patients with diabetes [5].

HbA1c is a well-established risk factor for adverse diabetes-related outcomes [6], and both fasting and postprandial hyperglycaemia are also major risk factors for diabetic complications [5]; however, data suggest that PPG excursions may represent a particularly relevant therapeutic target in patients with diabetes. Compared with long-term, sustained hyperglycaemia, BG fluctuations postprandially or during glucose 'swings' have a more specific triggering effect on oxidative stress, a factor that plays a pivotal role in the pathogenesis of various diabetic complications [7]. There is also evidence that postprandial hyperglycaemia is a greater predictor of cardiovascular disease than elevated FPG levels [8].

Rapid-acting insulin analogues (RAIAs; aspart, glulisine and lispro) and regular human insulin (RHI) have substantially different pharmacokinetic and pharmacodynamic profiles. Compared with RHI, RAIAs more closely mimic normal mealtime insulin excursions and their pharmacodynamic profiles are more similar to that of endogenous insulin (reviewed by Home [9]). The faster onset and offset of action [10], a lower incidence of hypoglycaemia [10], and less intrapatient variability on absorption [11] seen with RAIAs mean that these agents are preferred in patients with symptomatic hyperglycaemia, or those with glycaemia uncontrolled by basal insulin alone [10]. However, despite the advantages of RAIAs over RHI, both RAIAs and RHI are still widely used in the management of PPG excursions and GV in patients with diabetes $[10,12]$. The principal objective of the current meta-analysis is to update and consolidate the literature on this topic by using data from randomised controlled trials (RCTs) to address the impact of RAIAs and RHI on glycemic control, including PPG and long- and short-term GV [as measured by end-of-treatment (EOT) HbA1c and pre- to postprandial change in BG, respectively], in patients with type 1 diabetes (T1D) or type 2 diabetes (T2D).
Our hypothesis is that RAIAs are more effective than RHI in reducing PPG excursions and improving glycemic control in patients with T1D or T2D.

\section{METHODS}

This meta-analysis was conducted in accordance with the PRISMA guidelines for reporting of systematic reviews and meta-analyses (http:// www.prisma-statement.org/).

\section{Data Sources and Searches}

We searched PubMed for studies published between 1999 and 29 June 2016, using the following search string: 'insulin, short-acting' OR 'insulin lispro' OR 'insulin aspart' OR 'insulin glulisine' OR 'Novorapid' OR 'Apidra' OR 'Humalog' OR 'protamine suspension' OR 'insulin aspart protamine drug combination 30:70' OR 'Novolog' NOT ('pregnancy' OR 'pregnant women' OR 'hospitalization' OR 'institutionalization' OR 'paediatric' OR 'child' OR 'childhood' OR 'infant' OR 'newborn'). The search was then filtered to identify RCTs only.

The bibliographies of included studies and relevant reviews were searched to identify additional studies for inclusion.

\section{Study Selection}

We included all RCTs of patients with diabetes published from 1999 to 29 June 2016 that assessed the effects of RAIAs or RHI on glycemic control, with a focus on pre- and postprandial BG and HbA1c. All identified abstracts and study titles were initially reviewed independently by Antonio Nicolucci and Marco Orsini Federici. Subsequently, full articles of potentially appropriate trials were downloaded and screened for inclusion. The following article types and studies were excluded: reviews, editorials, case reports, clamp studies, studies of less than 4 weeks' duration, studies involving healthy volunteers, animal studies, and studies published in languages other than English.

Two reviewers (Antonio Nicolucci and Marco Orsini Federici) discussed and decided upon the 
final studies for inclusion in the meta-analysis. Only studies that reported both means and standard deviations (SDs) for the outcomes of interest were included in the final analysis.

\section{Data Extraction}

Data extraction was performed independently by Antonio Nicolucci and Marco Orsini Federici, who prepared a data extraction spreadsheet containing data collated from the final studies selected. Extracted data included study reference details, patient numbers, patient characteristics, measures of $\mathrm{GV}$, incidence of hypoglycaemia, and other relevant study information. Any discrepancies in the data gathered were discussed by Antonio Nicolucci and Marco Orsini Federici until a consensus was reached.

\section{Outcomes}

The primary outcomes were the mean differences between RAIAs and RHI at the end of the study in PPG $(\mathrm{mg} / \mathrm{dL})$, preprandial BG $(\mathrm{mg} / \mathrm{dL})$ and EOT HbA1c (\%). Outcomes for the sensitivity analysis were the mean differences between RAIAs and RHI at the end of the study regarding the difference between PPG and preprandial BG (mg/dL) and the change from baseline in HbA1c (\%).

\section{Data Synthesis and Analysis}

The meta-analysis of the effects of RAIAs versus RHI on mean pre- and postprandial BG levels and EOT HbA1c was conducted as follows: weighted mean differences and 95\% confidence intervals (CIs) between RAIAs and RHI were generated to yield the overall point estimate from the meta-analysis. Risk estimates from individual studies were pooled by using random- or fixed-effects models [13]. The metaanalysis did not adjust for covariates that may have affected the results of the included studies. When the $P$ value for heterogeneity was less than 0.10 , a random-effect meta-analysis was performed. For $P$ values of 0.10 or higher, a fixed-effects model was used. Heterogeneity across studies was assessed using the heterogeneity $\chi^{2}$ (Cochran Q) statistic and the $I^{2}$ test
[14]. These analyses were also performed using data showing the effects of RAIAs and RHI on the difference between postprandial and preprandial BG levels, and the difference between EOT and baseline HbA1c (sensitivity analyses). Risk of bias was not assessed.

All analyses were stratified according to the type of diabetes and were performed using a macro routine written in SAS language (SAS Release 9.4; Cary, NC, USA).

\section{Compliance with Ethics Guidelines}

This article is based on an analysis of previously conducted studies already in the public domain and does not include any studies with human participants or animals performed by any of the authors. Data were obtained via a published literature search.

\section{RESULTS}

\section{Literature Search Results}

Supplementary Fig. S1 shows the results of the literature search and study selection. Overall, 27 studies involving a total of 7452 randomised patients were included in the meta-analysis [15-41]. In terms of study design, 12 studies were parallel group [15, 16, 20, 25, 28, 29, 31, 33-37] and 15 were crossover studies (Supplementary Table S1) [17-19, 21-24, 26, 27, 30, 32, 38-41].

The majority of studies $(n=16)$ enrolled only patients with T1D $[15,16,18-22,24$, $28,29,32,36-38,40,41]$. Seven studies enrolled only patients with T2D [23, 25, 26, 31, 33, 35, 39], and four studies enrolled patients with T1D or T2D $[17,27,30,34]$. The studies varied in terms of the data reported for the endpoints of interest (Supplementary Table S2).

\section{Between-Group Differences in Pre- and Postprandial BG}

A total of nine studies (T1D, $n=7$; T2D, $n=1$; both T1D and T2D, $n=1$ ) reported the data needed to analyse differences between 
treatments in PPG or preprandial BG (eight and five studies, respectively) $[15,16,18,24,28-30$, $36,39]$.

The difference between RAIA- and RHI-treated patients in PPG was significant, in favour of RAIAs, in patients with T1D (mean betweengroup difference, $-22.2 \mathrm{mg} / \mathrm{dL} ; 95 \% \mathrm{CI}-27.4$ to $-17.0 \mathrm{mg} / \mathrm{dL} ; P<0.0001$; Fig. $1 \mathrm{a})$ but not in those with T2D (Fig. 1b). There was a moderate degree of heterogeneity across studies in both analyses (T1D: heterogeneity $\chi^{2}=9.03 ; P=0.17$; $I^{2}=34 \%$, 95\% CI 17-67\%; T2D: heterogeneity $\chi^{2}=1.80 ; P=0.18 ; I^{2}=44 \%, 95 \%$ CI $\left.18-100 \%\right)$.

Data for preprandial BG were only available in studies of patients with T1D $[15,16,24$, 30, 36]; in this analysis, which showed low heterogeneity across studies (heterogeneity $\chi^{2}=5.26 ; P=0.26 ; I^{2}=24 \%, 95 \%$ CI $\left.11-54 \%\right)$, there was a non-significant trend favouring RHI (Fig. 1c).

\section{Sensitivity Analysis}

Data for the analysis of between-group differences in change in BG following a meal (pre- vs PPG) were only available for patients with T1D (four studies) [15, 16, 24, 30]. In these patients, the difference in the change in BG was significant and favoured RAIAs (mean between-group difference, $-28.3 \mathrm{mg} / \mathrm{dL} ; 95 \%$ CI -52.6 to $-4.1 \mathrm{mg} / \mathrm{dL} ; P=0.022 ;$ Fig. 2 ). There was no evidence of heterogeneity across the studies in this analysis (heterogeneity $\chi^{2}=0.16 ; P=0.98$; $\left.I^{2}=0 \%\right)$.

\section{Between-Group Differences in HbA1c}

Eighteen studies reported the data required for analysis of HbA1c (T1D, $n=15$; T2D, $n=2$; T1D and T2D, $n=1)[15,16,18-22,24,25,28-30$, $32,36,37,39-41]$. In patients with T1D, the between-group difference in EOT HbA1c favoured RAIAs $(-0.13 \% ; 95 \% \mathrm{CI}-0.18$ to $-0.08 \% ; P<0.0001 ;$ Fig. 3a). In patients with $\mathrm{T} 2 \mathrm{D}$, the between-group difference was not significant (Fig. 3b). There was no evidence of heterogeneity across the studies in these analyses (T1D: heterogeneity $\chi^{2}=7.32 ; P=0.95$; $I^{2}=0 \%$; T2D: heterogeneity $\chi^{2}=1.08 ; P=0.58$; $\left.I^{2}=0 \%\right)$.

\section{Sensitivity Analysis}

The sensitivity analysis for HbA1c investigated the change in $\mathrm{HbA1c}$ from baseline to EOT with RAIAs and RHI; 15 studies (T1D, $n=13$; T2D, $n=1$; T1D and T2D, $n=1$ ) reported these data and were included in the analysis $[15,16,18,20,22,24,28-30,32,36,37,39-41]$. The between-group difference in the change from baseline in HbA1c was significantly in favour of RAIAs in both T1D $(-0.09 \%$; $95 \%$ CI -0.16 to $-0.03 \% ; P=0.006$; Fig. $4 \mathrm{a})$ and T2D $(-0.10 \% ; 95 \%$ CI -0.13 to $-0.07 ; P<0.0001$; Fig. 4b). Heterogeneity was high, and statistically significant, for the T1D analysis (heterogeneity $\chi^{2}=540.52 ; \quad P<0.0001 ; \quad I^{2}=100 \%$; 95\% CI $63-100 \%)$. In contrast, there was no evidence of heterogeneity between the two studies involved in the T2D analysis (heterogeneity $\left.\chi^{2}=0.26 ; P=0.61 ; I^{2}=0 \%\right)$.

\section{DISCUSSION}

The results of this meta-analysis, which incorporated 27 studies and more than 7000 patients, have shown that the use of RAIAs significantly reduced PPG compared with the use of RHI in patients with T1D. EOT HbA1c was also significantly lower with RAIAs than RHI in patients with T1D. Sensitivity analyses showed that the favourable results with RAIAs versus RHI were maintained when the difference between preand postprandial $B G$ and the change from baseline in HbA1c were analysed.

These results are in agreement with other studies comparing the effect of RAIAs versus RHI on PPG/glucose excursions in patients with T1D. These include a crossover study of 18 patients who received various rapid-/short-acting insulins, including insulin lispro, and RHI following stabilisation of preprandial glycaemia and before the ingestion of a standardised meal [42]. In this study the maximum postprandial glycaemia in the first $3 \mathrm{~h}$ post-injection was higher with RHI than with insulin lispro, and the area under the curve was also higher with RHI. These data demonstrate a reduction in postprandial glycemic excursions with insulin lispro versus RHI [42]. In a separate study involving 21 patients with T1D, a single-dose 


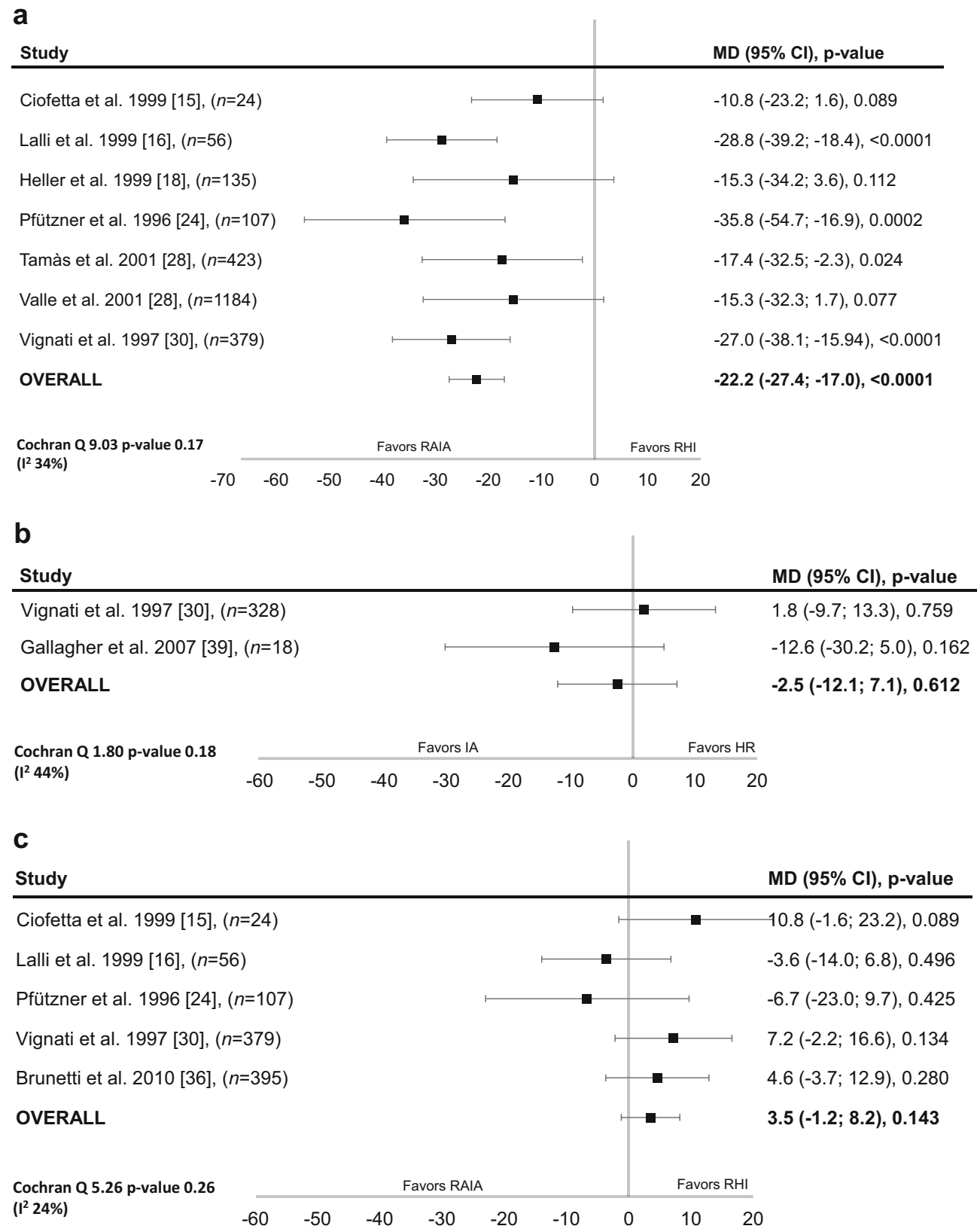

Fig. 1 Difference in blood glucose with rapid-acting insulin analogues and regular human insulin (fixed-effects model). Postprandial blood glucose in patients with a type 1 diabetes $(\mathrm{mg} / \mathrm{dL})$ or $\mathbf{b}$ type 2 diabetes $(\mathrm{mg} / \mathrm{dL})$.

administration of premeal insulin glulisine resulted in a lower maximum glucose excursion, a lower total BG exposure, and a lower maximum BG concentration compared with that seen after the administration of RHI [43]. These results confirm those of several older c Preprandial blood glucose in patients with type 1 diabetes $(\mathrm{mg} / \mathrm{dL})$. $C I$ confidence interval, $M D$ mean difference, RAIA rapid-acting insulin analogues, $R H I$ regular human insulin

studies in patients with T1D that showed a reduction in PPG/glucose excursions with RAIAs versus RHI [27, 44-46].

In the current meta-analysis, the number of studies involving patients with T2D was insufficient to allow us to draw valid conclusions; 


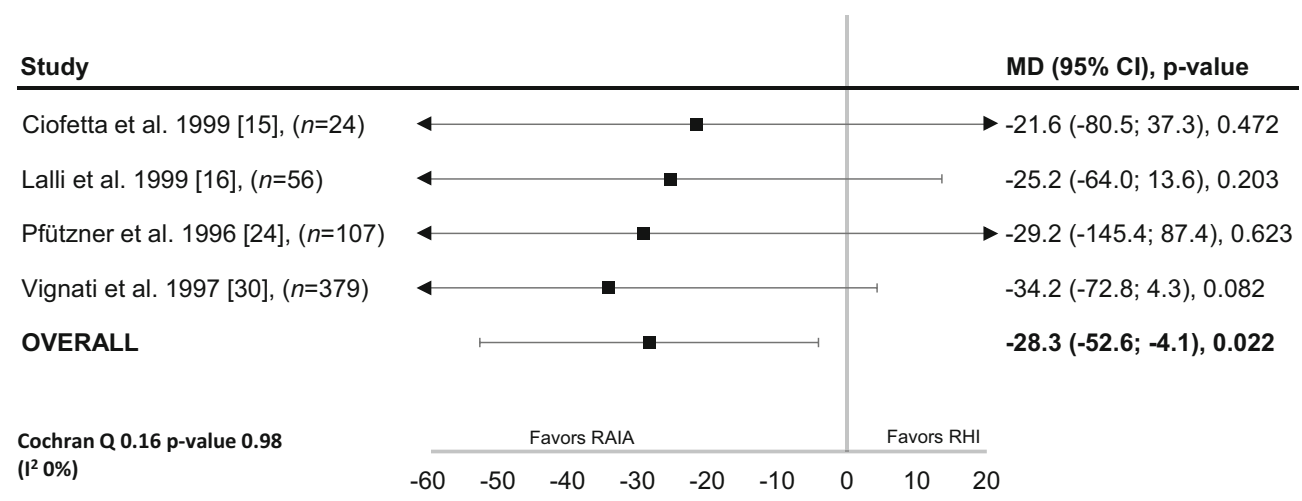

Fig. 2 Difference in the change in blood glucose following a meal (pre- vs postprandial; $\mathrm{mg} / \mathrm{dL}$ ) with rapid-acting with type 1 diabetes (fixed-effects model). CI confidence interval, $M D$ mean difference, $R A I A$ rapid-acting insulin insulin analogues and regular human insulin in patients analogues, $R H I$ regular human insulin

a

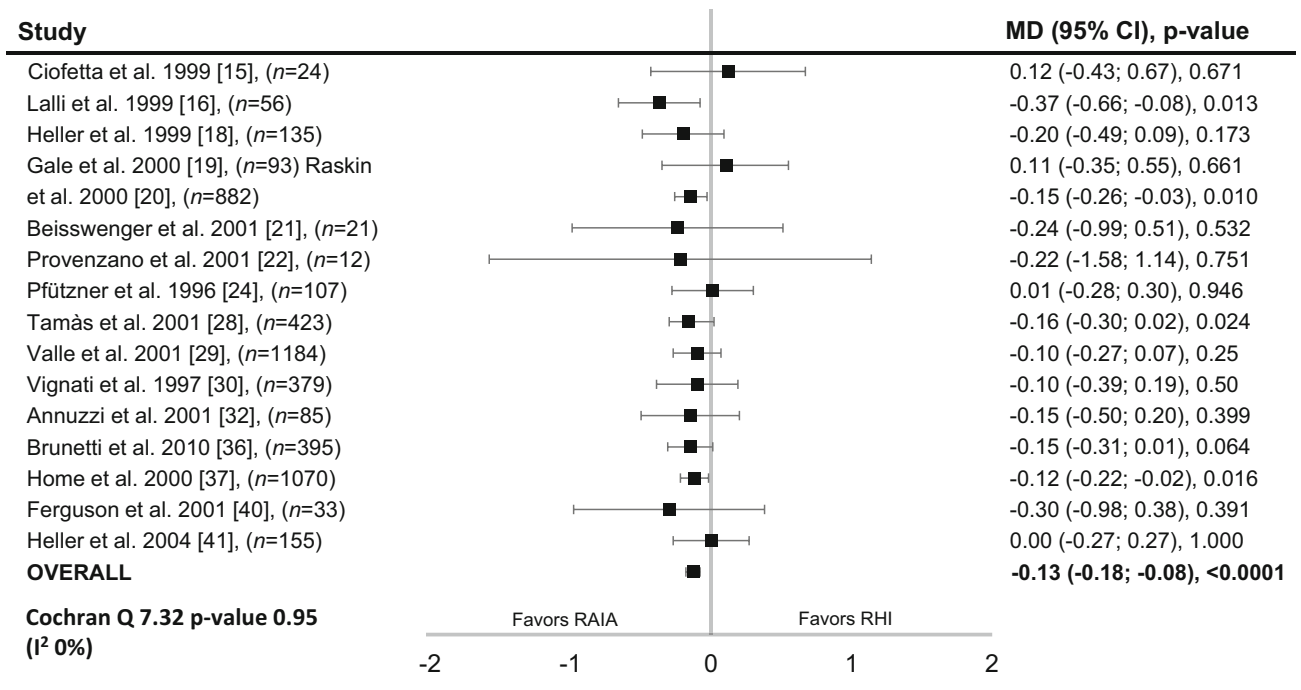

b

\begin{tabular}{l} 
Study \\
\hline Rayman et al. 2007 [25], $(n=890)$ \\
Vignati et al. 1997 [30], $(n=328)$ \\
Gallagher et al. 2007 [39], $(n=18)$ \\
OVERALL \\
Cochran Q 1.08 p-value $\mathbf{0 . 5 8}$ \\
$\left(\mathbf{I}^{2} \mathbf{0 \% )}\right.$
\end{tabular}

Fig. 3 Difference in glycated haemoglobin with rapidacting insulin analogues and regular human insulin. Endof-treatment glycated haemoglobin (\%) in patients with a type 1 diabetes or $\mathbf{b}$ type 2 diabetes (both fixed-effects

however, we would expect the results to be similar to those seen in patients with T1D given the available literature on the topic. For
MD (95\% Cl), p-value

$0.06(-0.06 ; 0.18), 0.333$

$0.000(-0.30 ; 0.30), 1.000$

$-0.13(-0.48 ; 0.22), 0.462$

$0.03(-0.07 ; 0.14), 0.531$

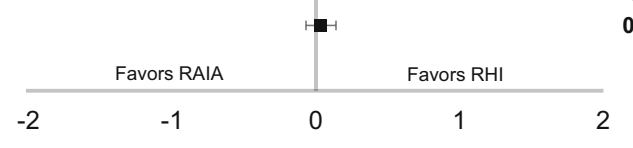

model). $C I$ confidence interval, $M D$ mean difference, $R A I A$ rapid-acting insulin analogues, $R H I$ regular human insulin

example, a randomised, multicentre study of 29 patients with T2D who received insulin aspart or RHI at a dose aimed at achieving a PPG below 
a

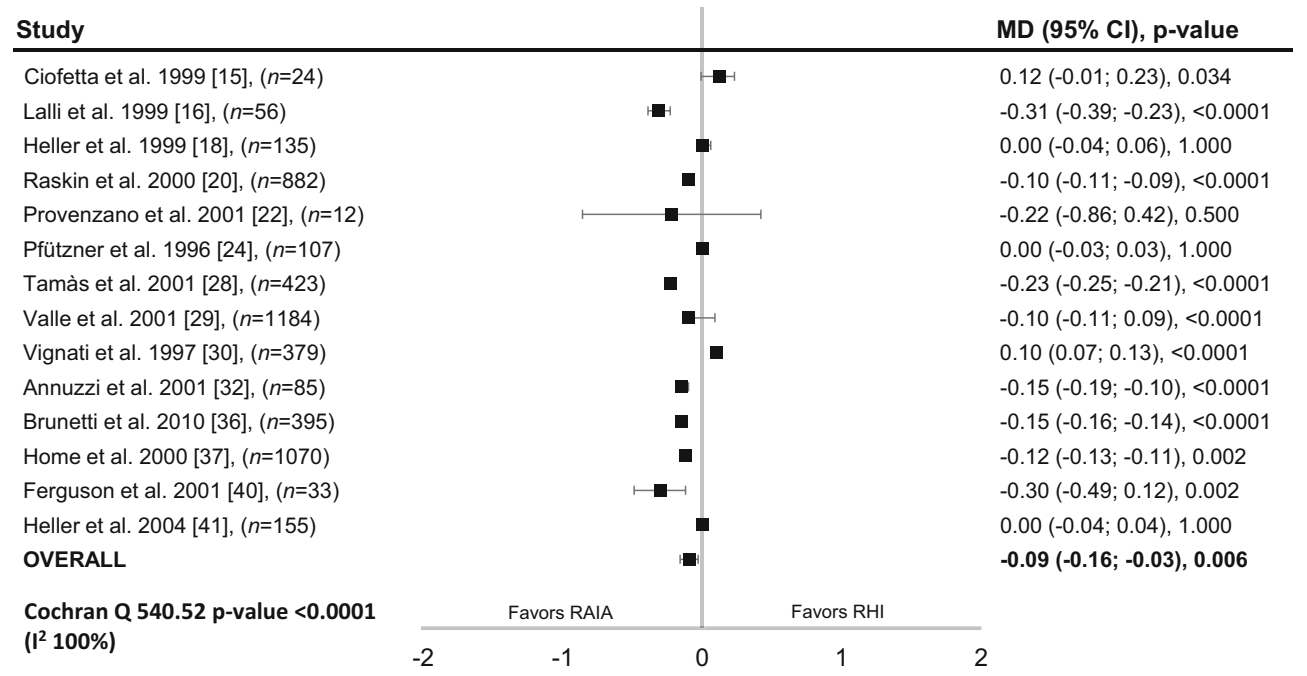

b

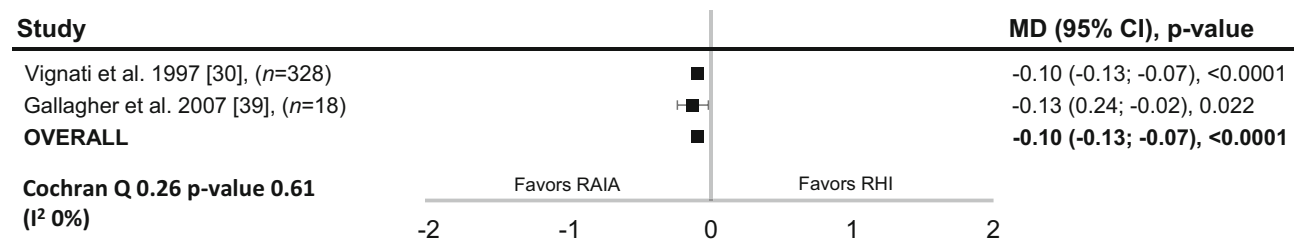

Fig. 4 Difference in glycated haemoglobin with rapidacting insulin analogues and regular human insulin. a Change from baseline in glycated haemoglobin (\%) in patients with type 1 diabetes (random-effects model); and

$140 \mathrm{mg} / \mathrm{dL}$ for 24 months showed that for the first 9 months of the study, patients receiving insulin aspart who had PPG levels of less than $140 \mathrm{mg} / \mathrm{dL}$ were on a significantly lower dose than those receiving RHI who achieved the same endpoint [47]. A randomised crossover trial involving 13 patients with $\mathrm{T} 2 \mathrm{D}$ who received insulin aspart or RHI demonstrated a significantly lower BG increase after a standardised meal with insulin aspart versus RHI [48]. Subanalyses of the $A_{1}$ chieve and IMPRO$\mathrm{VE}^{\mathrm{TM}}$ studies showed that switching from RHI to insulin aspart led to significant decreases in both HbA1c and PPG $[49,50]$.

In terms of estimation of GV, there is continued debate about the most suitable metrics for assessment of this outcome $[1,51]$. HbA1c is a measure of long-term, average glycemic control [51] and serial measurements of this metric can be used-as in our sensitivity analysis—as a b change from baseline in glycated haemoglobin (\%) in patients with type 2 diabetes (fixed-effects model). $C I$ confidence interval, $M D$ mean difference, $R A I A$ rapidacting insulin analogues, $R H I$ regular human insulin

marker of long-term GV [1, 12]. Short-term, within-day GV primarily reflects fluctuations between the fasted (preprandial) and fed (postprandial) states and can be assessed using data generated via self-monitoring of BG or continuous glucose monitoring [51]. In this analysis, we used the change in BG following a normal meal at home (pre- vs PPG) as an estimator of short-term GV in the sensitivity analysis. Both HbA1c and change in BG showed significant between-group differences in favour of RAIAs (change in HbA1c from baseline to EOT, T1D and T2D; change in BG following a meal, T1D). Since baseline HbA1c is a strong predictor of HbA1c response to insulin therapy [52-54], it was important to investigate whether potential differences in baseline HbA1c could have impacted the EOT HbA1c results obtained in our analysis. That the change from baseline in HbA1c still showed between-group differences 
in favour of RAIAs strengthens the main analysis results. Of the nine studies included in the analysis of preprandial BG, eight used neutral protamine Hagedorn insulin as the basal insulin, and one used insulin glargine; this lack of variation in the basal insulin used in the included studies prevented analysis of whether the choice of basal insulin affected preprandial glucose levels (and hence the change in pre- to postprandial BG level).

The outcomes assessed in the current analysis have additional value in the assessment of patients with diabetes: both FPG and PPG have been identified as major risk factors for complications of diabetes [5, 6], and PPG has been shown to independently correlate with HbA1c, making it a suitable marker for evaluating glycemic control. Although analysing the impact of decreased $\mathrm{GV}$ on adverse outcomes in patients with diabetes is beyond the scope of this analysis, it has been known for many years that chronic hyperglycaemia is associated with both macrovascular and microvascular complications [5-7]; therefore, agents that adequately control GV are also likely to control diabetes complications.

In some of the analyses reported here, the $\chi^{2}$ values, their associated $P$ values and the $I^{2}$ values indicated evidence of heterogeneity. This was particularly the case for the change in HbA1c from baseline to EOT in the patients with T1D $\left(\chi^{2}=540.52 ; P<0.0001 ; I^{2}=100 \%\right.$; 95\% CI 63-100\%) (Fig. 4a). However, the heterogeneity estimates reported herein, particularly those relating to patients with T2D, should be interpreted with caution; Cochrane's $Q$ (the $\chi^{2}$ statistic) has low power to reject the null hypothesis of homogeneity in meta-analyses involving small numbers of studies, and the $I^{2}$ statistic should also be interpreted cautiously because of its potential for bias in this situation [55]. The small number of studies included in many of the current analyses resulted from the absence of required data in many of the studies selected for inclusion. This and the heterogeneity of the studies are limitations of the present analysis. As a result, valid conclusions regarding the effect of RAIAs versus RHI on glycemic control in patients with T2D could not be drawn. Additional limitations of the study are that the meta-analysis was not adjusted for covariates, and a risk-of-bias analysis was not performed.

\section{CONCLUSIONS}

The results of this meta-analysis demonstrate that in patients with T1D, RAIAs are more effective at reducing postprandial glucose excursions and improving glycemic control than RHIs. More data are required to assess the effects of RAIAs and RHI on pre- and postprandial glucose levels, HbA1c and GV in T2D.

\section{ACKNOWLEDGEMENTS}

Funding. Sponsorship for this study and the journal's Rapid Service Fee were funded by Eli Lilly and Company.

Medical Writing Assistance. The authors would like to acknowledge Sheridan Henness, $\mathrm{PhD}$, and Janet Douglas, Vet $\mathrm{MB}, \mathrm{PhD}$ (Rx Communications) for medical writing assistance with the preparation of this article, funded by Eli Lilly and Company.

Authorship. All named authors meet the International Committee of Medical Journal Editors (ICMJE) criteria for authorship for this article, have provided critical revision of the manuscript for important intellectual content and have given their approval for this version to be published. All authors had full access to all of the data in this study and take complete responsibility for the integrity of the data and accuracy of the data analysis.

Authorship Contributions. AC was involved with the conception and design of the work, and the analysis and interpretation of the data. MOF was involved with the conception of the work, and the analysis and interpretation of the data. AN was involved with the conception and design of the work, and the acquisition, analysis and interpretation of the data. AC and PDB were 
involved with interpretation of the data for the work.

Disclosures. Antonella Corcos is a full-time employee and shareholder of Eli Lilly and Company. Marco Orsini Federici is a full-time employee and shareholder of Eli Lilly and Company. Antonio Nicolucci has received grants from Eli Lilly and Company, Novo Nordisk, Sanofi, Medtronic, and Astra Zeneca; and has received personal fees from Eli Lilly and Company, Novo Nordisk, Boehringer Ingelheim, and Astra Zeneca. Antonio Ceriello has been an advisory board member for Astra Zeneca, Boehringer Ingelheim, DOC Generici, Eli Lilly and Company, Janssen, Mundipharma, Novo Nordisk, and OM Pharma; has lectured for Astra Zeneca, Boehringer Ingelheim, Eli Lilly and Company, Mundipharma, Novartis, Novo Nordisk, Sanofi, and Takeda; and has received research grants from Astra Zeneca, Eli Lilly, Mitsubishi and Novartis. Paolo Di Bartolo has received grants and personal fees from Eli Lilly and Company, and NovoNordisk.

Compliance with Ethics Guidelines. This article is based on an analysis of previously conducted studies already in the public domain and does not include any studies with human participants or animals performed by any of the authors. Data were obtained via a published literature search.

Data Availability. All data generated or analysed during this study are included in this published article/as supplementary information files.

Open Access. This article is distributed under the terms of the Creative Commons Attribution-NonCommercial 4.0 International License (http://creativecommons.org/licenses/ by-nc/4.0/), which permits any noncommercial use, distribution, and reproduction in any medium, provided you give appropriate credit to the original author(s) and the source, provide a link to the Creative Commons license, and indicate if changes were made.

\section{REFERENCES}

1. Ceriello A, Monnier L, Owens D. Glycemic variability in diabetes: clinical and therapeutic implications. Lancet Diabetes Endocrinol. 2019;7(3): 221-30.

2. Monnier L, Colette C, et al. Glycemic variability: the third component of the dysglycemia in diabetes. Is it important? How to measure it? J Diabetes Sci Technol. 2008;2(6):1094-100.

3. Avignon A, Radauceanu A, et al. Nonfasting plasma glucose is a better marker of diabetic control than fasting plasma glucose in type 2 diabetes. Diabetes Care. 1997;20(12):1822-6.

4. Haddadinezhad S, Ghazaleh N. Relation of fasting and postprandial and plasma glucose with hemoglobinA1c in diabetics. Int J Diabetes Dev Ctries. 2010;30(1):8-10.

5. Monnier L, Colette C. Glycemic variability: should we and can we prevent it? Diabetes Care. 2008;31(Suppl 2):S150-4.

6. Stratton IM, Adler AI, et al. Association of glycaemia with macrovascular and microvascular complications of type 2 diabetes (UKPDS 35): prospective observational study. BMJ. 2000;321(7258):405-12.

7. Monnier L, Mas E, et al. Activation of oxidative stress by acute glucose fluctuations compared with sustained chronic hyperglycemia in patients with type 2 diabetes. JAMA. 2006;295(14):1681-7.

8. Monnier L, Lapinski $\mathrm{H}$, et al. Contributions of fasting and postprandial plasma glucose increments to the overall diurnal hyperglycemia of type 2 diabetic patients: variations with increasing levels of $\mathrm{HbA}(1 \mathrm{c})$. Diabetes Care. 2003;26(3):881-5.

9. Home PD. The pharmacokinetics and pharmacodynamics of rapid-acting insulin analogues and their clinical consequences. Diabetes Obes Metab. 2012;14(9):780-8.

10. Garber AJ, Abrahamson MJ, et al. Consensus Statement by the American Association of Clinical Endocrinologists and American College of Endocrinology on the Comprehensive Type 2 Diabetes Management Algorithm-2016 Executive Summary. Endocr Pract. 2016;22(1):84-113.

11. Madsbad S. Insulin analogues: have they changed insulin treatment and improved glycemic control? Diabetes Metab Res Rev. 2002;18(Suppl 1):S21-8.

12. Inzucchi SE, Bergenstal RM, et al. Management of hyperglycemia in type 2 diabetes, 2015: a patient- 
centered approach: update to a position statement of the American Diabetes Association and the European Association for the Study of Diabetes. Diabetes Care. 2015;38(1):140-9.

13. van Houwelingen HC, Arends LR, et al. Advanced methods in meta-analysis: multivariate approach and meta-regression. Stat Med. 2002;21(4):589-624.

14. Higgins JP, Thompson SG, et al. Measuring inconsistency in meta-analyses. BMJ. 2003;327(7414): 557-60.

15. Ciofetta M, Lalli C, et al. Contribution of postprandial versus interprandial blood glucose to HbA1c in type 1 diabetes on physiologic intensive therapy with lispro insulin at mealtime. Diabetes Care. 1999;22(5):795-800.

16. Lalli $\mathrm{C}$, Ciofetta $\mathrm{M}$, et al. Long-term intensive treatment of type 1 diabetes with the short-acting insulin analog lispro in variable combination with $\mathrm{NPH}$ insulin at mealtime. Diabetes Care. 1999;22(3):468-77.

17. Roach $\mathrm{P}$, Trautmann $\mathrm{M}$, et al. Improved postprandial blood glucose control and reduced nocturnal hypoglycemia during treatment with two novel insulin lispro-protamine formulations, insulin lispro mix25 and insulin lispro mix50. Mix50 Study Group. Clin Ther. 1999;21(3):523-34.

18. Heller SR, Amiel SA, et al. Effect of the fast-acting insulin analog lispro on the risk of nocturnal hypoglycemia during intensified insulin therapy. UK Lispro Study Group. Diabetes Care. 1999;22(10): 1607-11.

19. Gale EA. A randomized, controlled trial comparing insulin lispro with human soluble insulin in patients with type 1 diabetes on intensified insulin therapy. The UK Trial Group. Diabet Med. 2000;17(3):209-14.

20. Raskin P, Guthrie RA, et al. Use of insulin aspart, a fast-acting insulin analog, as the mealtime insulin in the management of patients with type 1 diabetes. Diabetes Care. 2000;23(5):583-8.

21. Beisswenger PJ, Howell SK, et al. alpha-Dicarbonyls increase in the postprandial period and reflect the degree of hyperglycemia. Diabetes Care. 2001;24(4): 726-32.

22. Provenzano C, Vero R, et al. Lispro insulin in type 1 diabetic patients on a Mediterranean or normal diet: a randomized, cross-over comparative study with regular insulin. Diabetes Nutr Metab. 2001;14(3):133-9.

23. Niskanen L, Jensen LE, et al. Randomized, multinational, open-label, 2-period, crossover comparison of biphasic insulin aspart 30 and biphasic insulin lispro 25 and pen devices in adult patients with type 2 diabetes mellitus. Clin Ther. 2004;26(4):531-40.

24. Pfützner A, Kustner E, et al. Intensive insulin therapy with insulin lispro in patients with type 1 diabetes reduces the frequency of hypoglycemic episodes. Exp Clin Endocrinol Diabetes. 1996;104(1):25-30.

25. Rayman G, Profozic V, et al. Insulin glulisine imparts effective glycemic control in patients with type 2 diabetes. Diabetes Res Clin Pract. 2007;76(2): 304-12.

26. Roach P, Yue L, et al. Improved postprandial glycemic control during treatment with Humalog Mix25, a novel protamine-based insulin lispro formulation. Humalog Mix25 Study Group. Diabetes Care. 1999;22(8):1258-61.

27. Skrha J, Smahelova A, et al. Insulin lispro improves postprandial glucose control in patients with diabetes mellitus. Sb Lek. 2002;103(1):15-21.

28. Tamàs $\mathrm{G}$, Marre $\mathrm{M}$, et al. Glycemic control in type 1 diabetic patients using optimised insulin aspart or human insulin in a randomised multinational study. Diabetes Res Clin Pract. 2001;54(2):105-14.

29. Valle D, Santoro D, et al. Italian multicentre study of intensive therapy with insulin lispro in 1184 patients with type 1 diabetes. Diabetes Nutr Metab. 2001;14(3):126-32.

30. Vignati L, Anderson JH Jr, et al. Efficacy of insulin lispro in combination with NPH human insulin twice per day in patients with insulin-dependent or non-insulin-dependent diabetes mellitus. Multicenter Insulin Lispro Study Group. Clin Ther. 1997;19(6):1408-21.

31. Abrahamian $\mathrm{H}$, Ludvik $\mathrm{B}$, et al. Improvement of glucose tolerance in type 2 diabetic patients: traditional vs. modern insulin regimens (results from the Austrian Biaspart Study). Horm Metab Res. 2005;37(11):684-9.

32. Annuzzi G, Del Prato S, et al. Preprandial combination of lispro and NPH insulin improves overall blood glucose control in type 1 diabetic patients: a multicenter randomized crossover trial. Nutr Metab Cardiovasc Dis. 2001;11(3):168-75.

33. von Bibra H, Siegmund $\mathrm{T}$, et al. Optimized postprandial glucose control is associated with improved cardiac/vascular function-comparison of three insulin regimens in well-controlled type 2 diabetes. Horm Metab Res. 2009;41(2):109-15. 
34. Boehm BO, Home PD, et al. Premixed insulin aspart 30 vs. premixed human insulin 30/70 twice daily: a randomized trial in type 1 and type 2 diabetic patients. Diabet Med. 2002;19(5):393-9.

35. Bretzel RG, Arnolds S, et al. A direct efficacy and safety comparison of insulin aspart, human soluble insulin, and human premix insulin (70/30) in patients with type 2 diabetes. Diabetes Care. 2004;27(5):1023-7.

36. Brunetti $\mathrm{P}$, Muggeo $\mathrm{M}$, et al. Incidence of severe nocturnal hypoglycemia in patients with type 1 diabetes treated with insulin lispro or regular human insulin in addition to basal insulin glargine. Nutr Metab Cardiovasc Dis. 2010;20(7):519-26.

37. Home PD, Lindholm A, et al. Insulin aspart vs. human insulin in the management of long-term blood glucose control in Type 1 diabetes mellitus: a randomized controlled trial. Diabet Med. 2000;17(11):762-70.

38. Colombel A, Murat A, et al. Improvement of blood glucose control in Type 1 diabetic patients treated with lispro and multiple NPH injections. Diabet Med. 1999;16(4):319-24.

39. Gallagher A, Butler TJ, et al. The effect of the optimal use of rapid-acting insulin analogues on insulin secretion in Type 2 diabetes. Diabetes Res Clin Pract. 2007;76(3):327-34.

40. Ferguson SC, Strachan MW, et al. Severe hypoglycaemia in patients with type 1 diabetes and impaired awareness of hypoglycaemia: a comparative study of insulin lispro and regular human insulin. Diabetes Metab Res Rev. 2001;17(4): 285-91.

41. Heller SR, Colagiuri S, et al. Hypoglycaemia with insulin aspart: a double-blind, randomised, crossover trial in subjects with Type 1 diabetes. Diabet Med. 2004;21(7):769-75.

42. Heinemann L, Hompesch $\mathrm{M}$, et al. Reduction of postprandial glycemic excursions in patients with type 1 diabetes: a novel human insulin formulation versus a rapid-acting insulin analog and regular human insulin. J Diabetes Sci Technol. 2011;5(3): 681-6.

43. Rave K, Klein O, et al. Advantage of premeal-injected insulin glulisine compared with regular human insulin in subjects with type 1 diabetes. Diabetes Care. 2006;29(8):1812-7.

44. Holleman F, Schmitt H, et al. Reduced frequency of severe hypoglycemia and coma in well-controlled IDDM patients treated with insulin lispro. The Benelux-UK Insulin Lispro Study Group. Diabetes Care. 1997;20(12):1827-32.
45. Raskin $\mathrm{P}$, Holcombe $\mathrm{JH}$, et al. A comparison of insulin lispro and buffered regular human insulin administered via continuous subcutaneous insulin infusion pump. J Diabetes Complications. 2001;15(6):295-300.

46. Anderson JH Jr, Brunelle RL, et al. Improved mealtime treatment of diabetes mellitus using an insulin analogue. Multicenter Insulin Lispro Study Group. Clin Ther. 1997;19(1):62-72.

47. Herrmann BL, Kasser C, et al. Comparison of insulin aspart vs. regular human insulin with or without insulin detemir concerning adipozytokines and metabolic effects in patients with type 2 diabetes mellitus. Exp Clin Endocrinol Diabetes. 2013;121(4):210-3.

48. Sourij H, Schmoelzer I, et al. Non-glycemic effects of insulin therapy: a comparison between insulin aspart and regular human insulin during two consecutive meals in patients with type 2 diabetes. Eur J Endocrinol. 2011;165(2):269-74.

49. El Naggar NK, Soewondo P, et al. Switching from biphasic human insulin 30 to biphasic insulin aspart 30 in type 2 diabetes is associated with improved glycemic control and a positive safety profile: results from the $\mathrm{A}(1)$ chieve study. Diabetes Res Clin Pract. 2012;98(3):408-13.

50. Shah S, Benroubi M, et al. Safety and effectiveness of biphasic insulin aspart 30/70 (NovoMix 30) when switching from human premix insulin in patients with type 2 diabetes: subgroup analysis from the 6-month IMPROVE observational study. Int J Clin Pract. 2009;63(4):574-82.

51. Kovatchev BP. Metrics for glycemic control-from HbA1c to continuous glucose monitoring. Nat Rev Endocrinol. 2017;13(7):425-36.

52. Home PD, Shen C, et al. Predictive and explanatory factors of change in HbA1c in a 24-week observational study of 66,726 people with type 2 diabetes starting insulin analogs. Diabetes Care. 2014;37(5):1237-45.

53. Liebl A, Jones S, et al. Clinical outcomes after insulin initiation in patients with type 2 diabetes: 6-month data from the INSTIGATE observational study in five European countries. Curr Med Res Opin. 2011;27(5):887-95.

54. Malek M, Aghili R, et al. Predictors of glycemic response and change in HbA1c following newly initiated basal insulin among insulin naive adults with type 2 diabetes. Acta Med Iran. 2017;55(9): 556-62.

55. von Hippel PT. The heterogeneity statistic I(2) can be biased in small meta-analyses. BMC Med Res Methodol. 2015;15:35. 